\title{
LA METÁFORA DEL LAZO SOCIAL EN JEAN-JACQUES ROUSSEAU Y ÉMILE DURKHEIM
}

\section{The social bond metaphor in Jean-Jacques Rousseau and Émile Durkheim}

\author{
Daniel Alvaro* \\ * CONICET, Universidad de Buenos Aires-IIGG (Argentina) \\ danielalvaro@gmail.com
}

Palabras clave

Lazo social

Individuo

Sociedad

Relación

Socialidad

\section{Keywords}

Social bond Individual

Society

Relation

Sociality

\begin{abstract}
Resumen
Este artículo es un aporte teórico para la comprensión de la metáfora del lazo social. En sentido amplio, esta remite a la unión entre los individuos y a las diferentes formas que asume la identidad colectiva ("Estado", "nación", "pueblo", etc.) en el seno de una sociedad determinada. En las últimas décadas ha llegado a convertirse en un tropo central en el ámbito de las ciencias sociales y las humanidades, aunque sus primeras apariciones se remontan a varios siglos atrás. En el artículo nos proponemos los siguientes objetivos: por un lado, analizar comparativamente los distintos usos y significados de esta metáfora en los discursos pioneros de Jean-Jacques Rousseau y Émile Durkheim — dos autores que además de haberla utilizado con frecuencia, le asignaron un papel fundamental en sus respectivas teorías-; y por otro lado, indagar en las implicaciones más generales de esta metáfora en tres planos de análisis diferenciados: el axiológico o valorativo, el epistemológico y el ontológico.
\end{abstract}

\section{Abstract}

This article is a theoretical contribution for the comprehension of the social bond metaphor. In a wide sense, social bond refers to the union among the individuals and the different forms of the collective identity ("Sate", "nation", "people", etc.) within a given society. In the last decades, it has become a central trope in the field of social sciences and the humanities even though its first apparitions date from many centuries ago. In this article we propose the following objectives: on the one hand, to analyze comparatively the different uses and meanings in the pioneer discourses of Jean-Jacques Rousseau and Émile Durkheim - two authors that, beyond using it frequently, they gave to this concept a fundamental role in their respective theories-; and, on the other hand, to inquire into more general implications of this metaphor in three differentiated analytical levels: the axiological or evaluative, the epistemological and the ontological.

Alvaro, D. (2017). La metáfora del lazo social en Jean-Jacques Rousseaux y Émile Durkheim. Papeles del CEIC, vol. 2017/1, papel 173, CEIC (Centro de Estudios sobre la Identidad Colectiva), UPV/EHU Press, http://dx.doi.org/10.1387/pceic.15726 
Entre las metáforas más sugerentes de la teoría social se encuentra la de "lazo social". Ella reúne en torno a sí figuras muy conocidas, incluidas aquellas que operan como conceptos fundamentales. Para tomar algunos ejemplos célebres, las nociones de "comunidad" y "sociedad", "grupo", "asociación" y "masa" son entendidas como formas diferenciadas del lazo social. Se trata, pues, de una expresión genérica, de uso muy extendido en el ámbito de las ciencias sociales y las humanidades, bajo la cual es posible reunir categorías heterogéneas y a veces incluso de significado o valor opuesto. En la misma medida, se trata de una expresión fuertemente polisémica, fluctuante en cuanto a su contenido, y por lo tanto, compleja desde el punto de vista de su definición y operacionalización con vistas a la investigación.

Antes de avanzar en la interpretación de sus principales usos, sus variadas connotaciones e implicaciones, se impone decir algo acerca del sentido general de este tropo. En su acepción mínima, es lo que une, lo que pone en relación a dos o más individuos. El lazo social es metáfora de la sociabilidad humana, es decir, de un modo particular de ser o estar con los demás, de un modo que tiende a la asociación antes que a la disociación y que supone de antemano individuos dispuestos a entablar relaciones con otros individuos, ya sea por inclinación natural, ya sea por necesidad o interés. El sesgo normativo es, en efecto, consustancial a la cuestión del lazo social y será objeto de análisis a lo largo de este artículo.

He aquí, esquemáticamente expuesto, el significado de esta metáfora y la razón por la cual no puede resultar más que sugerente para las disciplinas que tratan sobre lo social desde distintas perspectivas, visiblemente para la sociología, pero no en menor medida para la antropología, la filosofía política, la psicología y el psicoanálisis'

\footnotetext{
${ }^{1}$ ¿Acaso toda metáfora no es una especie de sugerencia o de insinuación, un rodeo con el cual se busca dar a entender algo o al menos facilitar su comprensión? De acuerdo con una interpretación tradicional que se remonta a la antigüedad clásica y, en lo fundamental, se mantiene vigente hasta la actualidad, la metáfora (del griego metaphorá: traslado, transporte) es una figura retórica que transfiere el sentido "literal" de los términos a un sentido "figurado" por medio de semejanza o analogía. La metáfora es el tropo que permite acceder al sentido "recto" de alguna cosa a través del sentido "desviado" expresado por otra cosa distinta de aquella. Aquí no sólo desconfiamos de la tranquilizadora oposición entre sentido literal o propio y sentido figurado, sino también de aquella otra entre discurso metafórico y discurso científico. Ante todo, porque no es seguro que al interior de un léxico determinado se pueda operar una diferenciación entre los términos llamados "naturales" y aquellos otros que se dicen "artificiales" o "derivados".
} 
La figura del lazo social reviste interés para nosotros por varios motivos. Entre otros, pero fundamentalmente, por ser uno de los tropos consagrados para designar la vida en común. Aquí, en primer lugar, esta figura será interrogada a partir de lo que se sugiere en los discursos de dos autores clásicos: Jean-Jacques Rousseau y Émile Durkheim. La elección de estos autores tiene buenas razones. La idea de lazo social es un tópico recurrente del pensamiento occidental desde tiempos remotos. A lo largo de la historia se sucedieron y muchas veces convivieron distintas representaciones del ligamen social, y algunas de ellas ya pueden ser rastreadas en la antigua filosofía griega (Rossi, 2015). Como es de esperar, estas representaciones se encuentran en estrecha relación con las concepciones del mundo que rigen cada época. De ahí que - para tomar un ejemplo concreto y lo suficientemente amplio- las imágenes del lazo producidas durante el periodo en que dominó la cosmovisión clásica de la filosofía política sean completamente distintas respecto de aquellas que se produjeron a partir del momento en que empezó a dominar la doctrina del derecho natural moderno. Ahora bien, dicho esto, hay que tener presente que el uso explícito del sintagma "lazo social" sólo aparece en las modernas teorías del contrato y más puntualmente en la obra de Rousseau. Poco más de un siglo después, Durkheim es uno de los autores que retoma esta expresión, en abierta discusión con la tesis de Rousseau, y le asigna un papel fundamental en su teoría sociológica. A través de Durkheim y de algunos de sus seguidores, esta marca léxica se convirtió en un locus classicus de los discursos contemporáneos sobre lo social. Su importancia para el debate actual radica en que además de mantener plena vigencia en el ámbito de las ciencias sociales y las humanidades, en los últimos tiempos su uso se acentuó y extendió más allá del campo estrictamente académico.

En segundo lugar, se procederá a un análisis de las implicaciones más importantes de la metáfora en cuestión. ¿Qué implica pensar la existencia en común en términoss de "Iazo"? ¿Lazo entre qué o quiénes?

Todas las palabras tienen una historia, pertenecen a una configuración y por lo tanto están ligadas a ciertos valores reconocibles que pueden ser rastreados a través de distintos métodos o estilos de trabajo. En cualquier caso, si subrayamos el carácter metafórico de la expresión "lazo social" no es para instalar la duda acerca de su estatuto cognoscitivo. Lo que está en juego en el estudio de esta metáfora no es su relación con el conocimiento ni su mayor o menor capacidad explicativa, sino aquello que el lazo social traslada en calidad de metáfora. 
¿Cuál sería el estatuto de los términos vinculados y del vínculo mismo? Tales son algunas de las preguntas a las que se intenta responder en este artículo.

\section{LAS CRISIS Del "LIEN SOCIAL": Rousseau, DuRkHeim y NOSOTROS}

En la actualidad, del mismo modo que en la época de Rousseau y luego en la de Durkheim, la pregunta por el lazo social incluye la inquietud por la existencia misma del vínculo $y$, consecuentemente, por aquellos fenómenos sociales que tienen incidencia positiva o negativa sobre la cohesión entre los individuos. Muy frecuentemente, allí donde se interroga el lazo social es porque directa o indirectamente se lo considera debilitado o en retirada. Referirse al lazo social equivale por lo general a tratar los problemas asociados a la amenaza siempre latente de la desintegración y de las distintas estrategias para superarla. En esos casos, la referencia viene acompañada de un diagnóstico sobre la crisis inminente o ya desencadenada del propio lazo. Aun cuando muchos de los discursos que lo nombran se inscriben en contextos socio-históricos diferenciados y siguen derroteros teóricos divergentes, es posible identificar una lógica del lazo social. En cuanto figura privilegiada de la sociabilidad, vale decir, de la disposición natural o voluntaria a la asociación, encarna un ideal de unidad basado en la conformidad y concordia entre los individuos. La lógica del lazo social establece un modo muy determinado de entender en qué consiste o en que debería consistir la sociedad desde el punto de vista de los valores que la fundamentan. La presuposición generalizada según la cual el lazo social es un hecho en sí mismo positivo condiciona y está condicionada a su vez por la percepción pesimista de la historia que comparten muchos discursos sociológicos clásicos. En este sentido, afirmamos que la normatividad es intrínseca al problema del lazo social.

Hoy en día, cuando tantos diagnósticos coinciden en el desencanto generalizado respecto de la sociedad global, no sorprende que la pregunta se relance con fuerza renovada. Al igual que en la época que signó el nacimiento de los sistemas científico-sociales, en el presente vuelve a prevalecer una representación sombría del lazo social. Numerosos factores contribuirian a este escenario mundial desencantado. Entre otros, cabe destacar las grandes transformaciones económicas, políticas, técnicas y culturales que se desencadenaron a 
partir de la década de 1970 a escala mundial. Las consecuencias negativas y no deseadas de dichas transformaciones nos enfrentarian a una nueva crisis del lazo social. Una crisis que en más de un sentido se asemeja a la que postulaban las teorías pesimistas de fines del siglo XIX $y$ principios del $X X$, ya que sus efectos apuntan en mayor o menor medida a la radicalización del individualismo y redundan en un retroceso de la sociabilidad. Sin embargo, la crisis actual diferiría de la anterior por su mayor profundidad y sus alcances imprevisibles. Lo que hoy en día se percibe en estado crítico es el sentido de los valores, las creencias y las prácticas que fundan el orden social y hasta la posibilidad de reencontrar un significado o una dirección para la vida en común.

En semejante contexto, es previsible que la vieja pregunta acerca de qué mantiene unidos a los individuos cobre nuevamente interés. Eso es lo que está sucediendo entre investigadores y especialistas, pero también entre referentes de la política, intelectuales y periodistas de un amplio espectro ideológico. Según Pierre Bouvier, aunque la expresión "lazo social" circula desde los tiempos de Rousseau, su utilización se ha vuelto habitual solamente desde fines del siglo $X X$ en paralelo a la proliferación de discursos que afirman la disolución de la realidad que aquella representa (2005: 245 y ss). A tal punto que, al menos en lengua francesa, la propagación de la misma llegó a considerarse omnipresente, incluso invasiva (Genestier, 2006). A pesar del uso más que extendido de la expresión, es sintomático de su equivocidad que esta no figure en prácticamente ninguna de las grandes enciclopedias ni en los diccionarios de ciencias sociales de referencia habitual (Bouvier, 2005: 28-30). Leslie Belton-Chevallier llegó a elaborar un listado de diccionarios de sociología donde el término no aparece para dar cuenta "del 'silencio' que reina en torno del 'lazo social'" (2009: 29). A este hecho, de por sí significativo, hay que agregar que son escasas las investigaciones dedicadas a problematizar esta cuestión ${ }^{2}$.

Para todo lo que sigue es necesario tener en cuenta que "lazo social" es sólo una traducción posible de lo que Rousseau y tantos otros después de él denominaron lien social. Otra traducción de este sintagma, también muy utilizada pero menos frecuente que la anterior, es la de

\footnotetext{
${ }^{2}$ Además de la bibliografía movilizada a lo largo de este artículo, quisiéramos destacar el notable libro de Godicheau y Sánchez León (2015) consagrado a analizar la "semántica histórica sobre el vínculo social", desde Platón hasta Heidegger, pasando por Maquiavelo y Hobbes, entre muchos otros.
} 
"vínculo social". Sucede que el sustantivo francés lien posee varios significados. Una primera acepción, que coincide con el sentido concreto de la palabra, es la de objeto flexible y alargado que sirve para enlazar o atar varias cosas entre sí. Una segunda acepción, también concreta, remite más generalmente al objeto de cualquier tipo que cumple la función de ligar distintos elementos. Una tercera acepción, en este caso abstracta o en sentido figurado, puede remitir o bien a la relación lógica percibida por un sujeto entre diversos hechos o ideas, o bien a lo que une a personas, estableciendo entre ellas relaciones sociales, morales, afectivas, etc. La palabra tiene aún otros significados más específicos pero todos ellos terminan por remitir a una $u$ otra de las opciones propuestas (TLFi - Trésor de la Langue Française informatisé).

Uno de los problemas que se presentan cuando se intenta traducir el sintagma lien social es que si bien su sentido es invariablemente metafórico, la mayoría de los autores tienden a tratarlo como si fuera un objeto material, vale decir, una cosa. De ahí que las traducciones para lien basculen entre "vínculo", palabra cuyo sentido es claramente inmaterial, y "lazo", que ocasionalmente puede referir a la unión entre personas pero cuyo significado más corriente es el de objeto físico ${ }^{3}$.

Cuando en El contrato social Rousseau nombra el lien social, se refiere al interés común del cual depende la existencia de la sociedad, aquel que resulta del acuerdo entre los intereses particulares y opuestos de los muchos individuos. Y el interés común - sea como sea que se lo interprete- no puede más que ser espiritual o moral. Sin embargo, Rousseau habla muy claramente de la solidez y del relajamiento, de la extensión y hasta del riesgo que aquel corre de romperse. Es interesante notar que prácticamente todas las apariciones de esta metáfora en el texto de Rousseau tienen una connotación material. Pero sobre todo es interesante notar que, después de Rousseau y más allá de él, la metáfora del "lazo" o del "vínculo social" envuelve esta ambigüedad entre la materialidad y la idealidad, lo concreto y lo abstracto, lo literal y lo figurado. La envuelve e inmediatamente queda envuelta en ella. La arrastra consigo mucho más allá de los confines históricos y teóricos de su contexto de emergencia. De hecho, la misma ambigüedad vuelve a

\footnotetext{
${ }^{3}$ Nótese que sólo tres de las trece acepciones de "lazo" ofrecidas en el Diccionario de uso del español de María Moliner, tienen un sentido moral. Más elocuente aún es el caso del Diccionario de la Real Academia Española, cuya definición de esta palabra no contempla más que acepciones materiales.
} 
aparecer en Durkheim, cuya perspectiva sobre el lien social confronta abiertamente con la de Rousseau y, bastante más cerca de nosotros, se la vuelve a encontrar en algunos de los autores contemporáneos que advierten sobre la crisis del lazo/vínculo social ${ }^{4}$.

Se dirá, quizás, que esta no es más que una forma de hablar o de escribir, un asunto de estilo o de retórica. Apenas "una forma de decir", según la fórmula utilizada para justificar el sentido metafórico de una expresión allí donde causa sorpresa o resulta inadmisible. Sin duda lo es. No obstante, se intentará mostrar cómo en el uso de esta figura aparecen comprometidas una serie de cuestiones de índole axiológica, epistemológica y ontológica íntimamente relacionadas con el modo habitual en que el pensamiento social concibe el ser o el estar en común.

\section{LAZO, VÍNCULO, NUDO}

Como indicábamos más arriba, Rousseau es un precursor en el uso de la metáfora examinada. En las primeras páginas de El contrato social (1762) ya encontramos una aproximación indirecta pero muy sugerente a la cuestión del lazo social. En el Capítulo II, titulado "Las primeras sociedades", Rousseau afirma que entre todas las asociaciones o sociedades, la familia no sólo es la más antigua sino la única que exhibe un lazo natural entre sus miembros. Y si, una vez roto el lazo, estos "continúan unidos, ya no es de manera natural, sino voluntariamente, y la familia misma sólo se mantiene por convención" (1993: 4). Veremos que entre los miembros de una sociedad política, muy por el contrario, existe un lazo de tipo social, es decir, ni natural ni necesario, sino voluntario ${ }^{5}$.

Es al comienzo del Libro Segundo, en el Capítulo I titulado "La soberanía es inalienable", donde Rousseau explica qué entiende por lazo social. A lo largo del Libro Primero establece algunos de los principios de su doctrina política, empezando por los más elementales. Es también en el Libro Primero donde expone su teoría del pasaje del estado de naturaleza al estado civil a través del pacto social.

El libro Segundo empieza con la exposición de "[l]a primera y más importante consecuencia de los principios anteriormente establecidos",

\footnotetext{
${ }^{4}$ Ver, por ejemplo, Paugam (2008).

${ }^{5}$ Para un estudio de las distintas modalidades del lazo y de los sentimientos que les corresponden en la obra de Rousseau, ver Lepan (2014).
} 
a saber, que "la voluntad general puede dirigir por sí sola las fuerzas del Estado, de acuerdo con la finalidad de su institución, que es el bien común; porque si la oposición de los intereses particulares ha hecho necesario el establecimiento de las sociedades, el acuerdo de estos mismos intereses es lo que lo ha hecho posible" (ibídem: 25). Según esta explicación, la fundación de las sociedades surge de la necesidad de encontrar un punto en común entre los intereses individuales contrapuestos, y este punto en común, a su vez, es condición de posibilidad de las sociedades. El estado propiamente social adviene, según Rousseau, cuando la conservación y la existencia misma del género humano en el estado natural se encuentran amenazadas. "Es lo que hay de común en estos diferentes intereses lo que forma el vínculo social [lien social]; y si no existiese un punto en el cual se pusiesen de acuerdo todos ellos, no podría existir ninguna sociedad" (ibídem: 25). El vínculo social nace de las diferencias entre los individuos, pero una vez establecido garantiza la identidad y la unidad de los mismos a través de la fundación de la sociedad y el Estado. El vínculo social expresa el interés común donde convergen las voluntades particulares y sus respectivas preferencias en favor de la voluntad general, cuya tendencia natural - Rousseau lo repite una y otra vez a lo largo del tratado- es la "igualdad", la "utilidad pública", la "rectitud" y la "justicia". Por todo lo dicho hasta acá, es evidente que no puede alterarse el lazo social sin que se altere simultáneamente el cuerpo social y político que de aquel depende. He aquí la importancia decisiva de esta noción al interior del proyecto teórico rousseauniano.

Cuando la solidez del lazo social comienza a erosionarse se pone en riesgo toda la arquitectura sociopolítica puesto que esta depende directamente de aquella. Los motivos que pueden llevar al rompimiento del lazo social se deducen fácilmente de las premisas relativas a su formación y establecimiento. Cuando el interés común es reemplazado por el interés individual el lazo social sucumbe $y$, con él, sucumben las instituciones que lo tienen por fundamento. En palabras de Rousseau:

“(...) cuando el nudo social comienza a aflojarse y el Estado a debilitarse, cuando los intereses particulares empiezan a adquirir fuerza y las pequeñas sociedades a influir sobre la grande, el interés común se altera y encuentra oposición; ya no reina la unanimidad en las votaciones; la voluntad general ya no es la voluntad de todos; surgen contradicciones, debates, y la mejor opinión no pasa sin discusión. 
Finalmente, cuando el Estado, próximo a su ruina, no subsiste sino por una fórmula ilusoria y vana; cuando el vínculo social se ha roto en todos los corazones; cuando el más vil interés se ampara descaradamente bajo el nombre sagrado del bien público, entonces la voluntad general enmudece y todos, guiados por motivos secretos, dejan de opinar ya como ciudadanos, como si el Estado no hubiese existido jamás, y se hacen pasar falsamente por leyes, decretos inicuos, que no tienen más finalidad que el interés particular" (ibídem: 104).

Evidentemente, para Rousseau, el estado del vínculo social es un indicador insustituible de la salud del cuerpo colectivo. La solidez y la tensión del lazo social -o del "nudo" (ncud), como también lo llama, redoblando así la ambigüedad característica de toda esta metafóricason signos del vigor del orden social y político. Mientras que su relajamiento $y$, en el límite, su ruptura, son signos inequivocos de la decadencia física y moral a la que queda expuesto el cuerpo del pueblo. Una vez roto el "enlace" (liaison) que mantenía a los individuos unidos entre sí, el todo se dispersa en partes cuyos intereses se vuelven irreconciliables y el noble interés de la comunidad queda reducido a viles intereses individuales. Sólo un lazo social sano puede garantizar el predominio real y efectivo de lo universal sobre lo particular. De ahí la insistencia de Rousseau tanto en la dureza como en la duración del lazo. Ambas características se encuentran relacionadas aunque dependen de condiciones diferentes. Por ejemplo, entre los factores que hacen a la fortaleza del vínculo social, además de los ya mencionados, Rousseau incluye la extensión que puede alcanzar un Estado. "Mientras más se extiende el vínculo social, más se afloja, y, en general, un Estado pequeño es proporcionalmente más fuerte que uno grande" (ibídem: 4546). Entre los factores que influyen sobre la duración del vínculo hay que contar muy especialmente aquellos que están relacionados con el ejercicio legítimo del poder por parte de la autoridad soberana, en el sentido de que los vínculos serán más duraderos en la medida en que el poder se ejerza de manera legítima (lo que equivale a decir, conforme a las leyes del Estado resultante del pacto social), y pasajeros o incluso efímeros en caso contrario.

Ahora bien, tanto o más importante que distinguir entre un lazo "duradero" y otro "pasajero", es distinguir entre lo que Rousseau considera como "falsas nociones sobre el lazo social" y aquella que estima verdadera. Esta última distinción la encontramos desarrollada en 
el así llamado Manuscrito de Ginebra, que no es otra cosa que la primera versión de El contrato social. Publicado póstumamente, se conjetura que el Manuscrito fue redactado entre 1755 y 1761. Se trata de un bosquejo cuyo contenido difiere en varios puntos respecto de la versión definitiva. Aquí nos interesa especialmente el Capítulo 5 del Libro I del Manuscrito, titulado "Falsas nociones sobre el lazo social". El mismo no figura en la versión definitiva de El contrato social.

Dicho capítulo comienza con la siguiente afirmación: "Hay mil maneras de juntar a los hombres, pero sólo una de unirlos. Por eso, en esta obra no ofrezco más que un método para la formación de las sociedades políticas" (Rousseau, 2004: 564-565). Los hombres, cuya condición natural parece ser la dispersión, pueden ser reunidos por distintos mecanismos, todos ellos falsos en la medida en que no produzcan una verdadera unión. Para Rousseau, la noción verdadera de lazo social es aquella capaz de unir a los hombres, y a su vez, una unión semejante sólo es posible en una sociedad política que sea el resultado del contrato social. Como explica la autora del estudio preliminar, de los comentarios y la traducción del Manuscrito, Vera Waksman, "[e]n este texto, Rousseau no se ocupa del problema del origen de las sociedades políticas, sino de su legitimidad" (2004: 564-565, nota 46). De la cuestión del origen de la sociedad humana se ocupa en otro capítulo del Manuscrito sobre el que diremos algo a continuación. Antes, es preciso constatar que las falsas nociones sobre el lazo social se corresponden con "otras vías de asociación civil", básicamente con aquellas que promueven Grocio y Hobbes. El análisis de las mismas será retomado en la versión definitiva de El contrato social. La verdadera noción del lazo, "el lazo social de la Ciudad", es la única que da lugar a un poder legítimo, entendiendo por poder legítimo todo Estado que tenga por objeto el bien común y no sólo el de unos pocos.

Otra diferencia notable sobre la que quisiéramos llamar la atención es un nuevo capítulo que desaparece de una versión a la otra y del que prácticamente no quedan huellas. Se trata del Capítulo 2 del Libro I, titulado "De la sociedad general del género humano". Allí, Rousseau polemiza con un artículo de Diderot titulado "Derecho natural" (1755) que había sido publicado en la Enciclopedia. El tema de este capítulo es la idea misma de sociabilidad. La pregunta que desencadena el análisis es "de dónde nace la necesidad de las instituciones políticas" (ibídem: 550). Rousseau comienza a responder a través de un rodeo. Su hipótesis 
es que los hombres del estado primitivo eran independientes unos de otros y así se mantuvieron mientras la fuerza de cada uno era proporcional a sus necesidades. Sólo desde el momento en que las necesidades aumentaron, los hombres comenzaron a asociarse para satisfacer deseos ilimitados: "nuestras necesidades nos acercan [unos a otros] a medida que nuestras pasiones nos dividen; y cuanto más enemigos somos de nuestros semejantes, menos podemos prescindir de ellos" (ibídem: 550). Este primer acercamiento está signado por la situación de dependencia, no por la voluntad. Los hombres no eligen libremente contar con los demás, sino que ya no pueden prescindir de ellos a causa de su codicia. "Tales son los primeros lazos de la sociedad general" (ibídem: 550). Lo que Rousseau llama aquí "primeros lazos" y "sociedad general" está muy alejado de su concepción de la sociabilidad. En primer lugar, estos primeros vínculos son forzados y están basados en el puro interés individual. En segundo lugar, la sociedad a la que dan lugar es asimétrica, anómica e inestable, además de injusta y por demás ilusoria desde el punto de vista de la unión que ella debería hacer efectiva. La necesidad de las instituciones políticas nace de una situación social que Rousseau encuentra deplorable ${ }^{6}$.

No hay que perder de vista que las afirmaciones con las que comienza este capítulo están destinadas a refutar la noción de "identidad de naturaleza" planteada por Diderot en "Derecho natural". La posición de Rousseau es muy clara. A diferencia de lo que afirma Diderot, los hombres no tienden a identificarse entre sí de manera espontánea; aquello que los reúne en sociedad no es lo que naturalmente tienen en común, sino más bien sus necesidades y deseos particulares. Desde la perspectiva rousseauniana no existe nada parecido a una sociabilidad natural: "ese presunto tratado social, dictado por la naturaleza, es una

\footnotetext{
${ }^{6}$ En el Manuscrito de Ginebra Rousseau traza una imagen sórdida del estado primitivo que contrasta con el cuadro idílico que de ese mismo estado nos pinta en el Discurso sobre el origen y los fundamentos de la desigualdad entre los hombres (1754). En cualquier caso, dicho contraste no modifica el tono entre nostálgico y melancólico con que el autor, aquí y allá, se refiere a la felicidad inherente a la condición originaria del hombre. Condición que en el Discurso tenía una existencia hipotética y que en el Manuscrito adopta más bien la forma de un mito: "la paz y la inocencia se nos han escapado para siempre, antes de que hubiéramos saboreado sus delicias. Imperceptible para los estúpidos hombres de los primeros tiempos, desvanecida para los hombres esclarecidos de los tiempos posteriores, la vida feliz de la edad de oro fue siempre un estado ajeno a la raza humana, bien por haberla desconocido cuando podía gozar de ella, bien por haberla perdido cuando habría podido conocerla" (2004: 551-552).
} 
verdadera quimera" (ibídem: 552). La "sociedad general" está a medio camino entre el estado de naturaleza y el estado civil. Los hombres pueden vivir juntos sin por ello estar unidos. La asociación legítima de la cual depende la justicia y la igualdad, la virtud y la felicidad se basa en la unión de las partes y el todo. La verdadera sociabilidad es completamente artificial. Otra forma de decir que "el lazo no está dado, sino que es instituido" (Bernardi, 2006: 480). Al final del capítulo, Rousseau llama expresamente a corregir "la falla de la asociación general", a perfeccionar "el arte" de instituir una asociación verdadera (2004: 556-557). El lazo social es la unión auténtica, sólida y duradera entre individuos que se han impuesto la tarea conjunta de acordar sus intereses individuales a fin de que pueda existir una sociedad y un Estado que vele por el bien común. Digamos para concluir que, desde este punto de vista, el lazo social no sólo es posterior a la existencia de los individuos independientes y libres, sino también a la existencia misma de ese orden en parte natural y en parte social que recibe el nombre de sociedad o asociación general. Cuestión que nos lleva a una vieja pregunta a la que desde muy temprano se vio sometida la lógica del contrato: ¿cómo es posible que la construcción necesariamente social del lazo, vínculo, nudo o como quiera que se llame a la "unión real entre los individuos" tenga lugar en un orden, al que según Rousseau, debemos considerar de hecho y de derecho como pre-social?

Por cierto, esta pregunta no pasó desapercibida para Durkheim, quien fue un destacado lector y comentarista de la obra de Rousseau. La influencia de uno sobre otro está fuera de discusión. No obstante, hay diversas interpretaciones sobre el alcance de esta impronta y sobre los puntos de convergencia y divergencia entre ambos ${ }^{7}$. Aquí no abordaremos de lleno ni en detalle esta cuestión, sino que en principio nos limitaremos a comentar algunos pasajes de Durkheim sobre Rousseau a propósito del lazo social.

Un conocimiento somero de los fundamentos que sostienen las teorías de estos autores alcanza para entrever los puntos en los que se distancian. Desde sus primeros textos, Durkheim critica el trasfondo individualista del pensamiento de Rousseau, sobre todo la idea de que el hombre es un ser solitario por naturaleza. Asimismo, critica la idea de

\footnotetext{
${ }^{7}$ En su famoso libro sobre Durkheim, Steven Lukes sienta su posición y asimismo se distancia de otras interpretaciones sobre la relación Rousseau-Durkheim, como por ejemplo la de Sheldon Wollin. Ver Lukes (1984: 282-287).
} 
que la sociedad es un orden artificial que depende de la voluntad de asociación de los individuos. Sin embargo, estas críticas van a ser amenizadas y en parte neutralizadas en escritos posteriores donde Durkheim le reconoce a Rousseau una concepción de los hechos sociales específica y a la vez diferenciada de la de los hechos individuales ${ }^{8}$. Durkheim cuestiona claramente la comprensión rousseauniana del lazo social. En un texto temprano, "La ciencia positiva de la moral en Alemania" (1887), compara el significado de las nociones de individuo y sociedad para los economistas de la Escuela de Manchester con el significado que tienen para Rousseau: "en el fondo los economistas liberales son discípulos inconscientes de Rousseau del que reniegan erróneamente. Reconocen, es cierto, que el estado de aislamiento no es lo ideal; pero como Rousseau, no ven en el lazo social más que un acercamiento superficial, determinado por confluencias de intereses" (1975a: 271-272).

En un texto apenas anterior, "Los estudios de ciencia social" (1886), llama la atención sobre el error de considerar la utilidad como el único lazo que nos une a nuestros semejantes: "si lo útil fuera el único lazo social, las sociedades no serían más que asociaciones de un día, ya que nada es más cambiante que el interés" (1970: 201). Para Durkheim hay causas completamente distintas que explican la asociación. "Existe, o al menos se forma en el curso de la evolución, una necesidad de sociabilidad $y$ de instintos sociales que son absolutamente desinteresados" (ibídem: 201). Ahora bien, aun cuando el origen del lazo social para Rousseau y Durkheim sea diferente, incluso opuesto, es preciso notar que una vez instaurado el vínculo, ambos autores apuestan a la solidez y a la constancia del mismo: "una sociedad cuyos miembros no estuvieran vinculados unos a otros por algún lazo sólido y durable parecería un montón de polvo desintegrado que el menor viento no tardaría nada en dispersar por todo el horizonte" (ibídem: 207). Por una parte, este pasaje recuerda la connotación material asociada a la metáfora del lazo que ya tuvimos oportunidad de confirmar en los textos de Rousseau. Con un vocabulario casi idéntico al de su predecesor, Durkheim también trata el lien social como si fuera un objeto o una cosa. Habla de lazos fuertes y débiles, estables y efímeros, profundos y superficiales; lazos que pueden ser más o menos resistentes y que dadas las circunstancias pueden tanto tensarse como aflojarse,

\footnotetext{
${ }^{8}$ Ver, en especial, Durkheim (2001). 
anudarse, desanudarse, quebrarse o romperse. Por otra parte, se puede ver aquí la activación de una serie de oposiciones binarias (por un lado, lo sólido y lo frágil, lo duradero y lo pasajero, por otro, la integración y la desintegración, la unión y la dispersión) que van a orientar el ideal durkheimiano de sociabilidad. Más adelante volveremos sobre esta cuestión.

Pero las diferencias con Rousseau a propósito del lazo social no terminan aquí. En una de sus Lecciones de sociología (1890-1900), Durkheim problematiza la noción de contrato y critica específicamente la teoría del contrato social:
"La noción de contrato suele ser considerada una operación tan simple que se la ha llegado a considerar como el hecho elemental del que derivarian todos los demás hechos sociales. La teoría del contrato social se basa en esta idea. El lazo social por excelencia, que une a los individuos en una misma comunidad, habría sido - o debería haber sido- producto de un contrato. Y si se hace del contrato un fenómeno primitivo, sea cronológicamente o sea - como lo entiende Rousseau- lógicamente, es porque la noción parece clara por sí misma. (...) Pero nada es más engañoso que esta claridad aparente. Lejos de que la institución del contrato sea primitiva, no aparece $-y$, sobre todo, no se desarrolla - sino en una fecha muy tardía. Lejos de ser simple, es de una extrema complejidad y no es fácil ver cómo se ha formado" (2003: 237).

El contrato no es simple, elemental ni primitivo. Es siempre el resultado de un estado de cosas o de personas, de una situación jurídica determinada que establece ciertos derechos y obligaciones. La sociedad no podría ser la consecuencia de un contrato, puesto que la institución contractual implica un cierto grado de desarrollo de la organización social. Del mismo modo: el lazo social no podría ser estipulado por contrato ya que para hablar o pensar en términos contractuales se necesita una sociedad constituida. Durkheim rechaza tanto la explicación voluntarista y utilitarista como la naturaleza contractual del lazo social. Ambos supuestos aparecen identificados principalmente con el pensamiento de Rousseau, aunque Durkheim también critica el individualismo en las teorias de Hobbes y Montesquieu.

Cabe preguntarse, entonces, cuál es la explicación sociológica del lazo social que se propone como alternativa. Durkheim comienza a elaborar una respuesta desde su primer curso de sociología dictado en 18871888. De hecho, entiende que "el problema inicial de la sociología" pasa 
por saber "cuáles son los lazos que unen a los hombres entre sí, es decir, lo que determina la formación de agregados sociales" (Durkheim, 1975b: 9). Desde la perspectiva durkheimiana, hablar de los lazos que unen a los hombres equivale a hablar de "solidaridad social". Este concepto fue objeto de un primer tratamiento en el curso introductorio de 1887-1888 -curso titulado, precisamente, "Solidaridad social"-, pero encontró un desarrollo más amplio en su célebre tesis convertida en libro: La división del trabajo social (1893).

En este libro se distinguen dos tipos de lazos sociales o solidaridades: la "solidaridad mecánica", que deriva de las similitudes y donde la personalidad colectiva se impone sobre la personalidad individual, y la "solidaridad orgánica", que deriva de la división del trabajo y donde aumenta la variabilidad individual. La tesis sobre las causas y consecuencias de la sustitución progresiva de la primera por la segunda es lo suficientemente conocida. Lo que nos interesa aquí no es la tesis en sí misma, sino lo que en este libro Durkheim entiende por lien social tanto cuando lo refiere a las sociedades tradicionales como cuando lo refiere a las sociedades modernas. Así, pues, lo que tienen en común los lazos sociales del pasado y los del presente, a pesar de sus enormes diferencias, es que todos ellos son lazos de solidaridad que aseguran la unión de las sociedades. En suma, la solidaridad favorece la "cohesión": "[a]llí donde es fuerte, inclina fuertemente a los hombres unos hacia otros, los pone frecuentemente en contacto, multiplica las ocasiones que tienen de encontrarse vinculados" (Durkheim, 2008: 144). Si dejamos de lado las formas sociales específicas que se corresponden con los distintos tipos de solidaridad social, sólo se puede captar lo que es común a todos ellos:

\begin{abstract}
“(...) a saber, la tendencia general a la sociabilidad, tendencia que es siempre y en todas partes la misma y que no está ligada a ningún tipo social en particular. Pero ese residuo no es más que una abstracción, pues la sociabilidad en sí no se encuentra en ninguna parte. Lo que existe y vive realmente son las formas particulares de la solidaridad, la solidaridad doméstica, la solidaridad profesional, la solidaridad nacional, la de ayer, la de hoy, etc." (ibídem: 146).
\end{abstract}

Visiblemente, Durkheim está interesado en privilegiar el punto de vista sociológico sobre el de los moralistas y psicólogos. La "sociabilidad en si" no sería más que una abstracción, una generalidad filosófica sin valor científico. Quizás. Sin embargo, Durkheim se equivoca o no es del todo 
veraz cuando da a entender que la sociabilidad, además de abstracta, es "algo demasiado indefinido" que permanece en "estado de indeterminación". Durkheim rechaza con argumentos sociológicos cualquier forma de esencialismo o sustancialismo, pero esto no significa que su aproximación a la cuestión carezca de una concepción muy determinada de la sociabilidad. Desde su perspectiva, "la tendencia general a la sociabilidad" es justamente la tendencia a la solidaridad. Durkheim presupone que el hombre es solidario por naturaleza, que la solidaridad es inherente al modo de ser humano, siempre y en todas partes. Como sociólogo, lo que le interesa es clasificar y comparar las diferentes especies de solidaridad social, para lo cual debe suplantar el hecho moral y por lo tanto inmaterial que es la solidaridad por un hecho externo que lo materialice -a saber, el derecho-, y examinar uno a través del otro. Eso es exactamente lo que hace pero siempre dando por supuesto que la solidaridad, es decir, la disposición a ser o estar unido con el resto de los individuos, es el fundamento de toda relación social. La solidaridad, para Durkheim, es en principio "positiva". Si bien reconoce una "solidaridad negativa", también explica que "[n]o se trata de una verdadera solidaridad, con una existencia propia y una naturaleza especial, sino más bien del lado negativo de todo tipo de solidaridad" (ibiem: 190). La solidaridad negativa es apenas una "emanación", una "repercusión", un "acompañamiento necesario" de la solidaridad verdadera. En ningún momento Durkheim desconoce la solidaridad negativa ni el tipo de relaciones que ella expresa. Pero se encarga de dejar en claro que esta siempre tiene lugar sobre un fondo de positividad. El magma social implica relaciones de cooperación, implica una afección mutua y desinteresada que no es exagerado llamar amor. Pues "para que los hombres se reconozcan y se garanticen mutuamente derechos, es necesario primero que se amen, que, por alguna razón, se estimen los unos a los otros y la sociedad de la que forman parte" (ibídem: 192).

La misma positividad fundamental le corresponde al lazo social. Este puede ser negativo, ciertamente, pero en este caso no se puede hablar de "un lazo social verdadero". El ejemplo más claro es la creencia contemporánea en torno al individuo, esa suerte de culto por la "dignidad de la persona" que, sin embargo, no llega a conformar un auténtico vínculo social. "Es de la sociedad de donde extrae todo lo que posee de fuerza, pero no es a la sociedad a lo que nos liga, sino a 
nosotros mismos. Por consiguiente, no constituye un lazo social verdadero" (ibídem: 239). Según este principio diferenciador, es verdadero el lazo de solidaridad que tiende a la integración de los individuos y que, por consiguiente, puede equipararse con las reglas morales. Durkheim reafirma esta idea central en la conclusión de La división del trabajo social: "El derecho y la moral son el conjunto de los lazos que nos unen unos a otros y con la sociedad, que hacen de la masa de los individuos un agregado y un todo coherente". Y, en relación con la moralidad, agrega inmediatamente: "Es moral (...) todo aquello que es fuente de solidaridad, todo lo que fuerza al hombre a contar con otro, a regular sus movimientos de acuerdo con algo más que los impulsos de su egoísmo, y la moralidad es tanto más sólida cuanto más numerosos y fuertes son estos lazos" (ibídem: 431) ${ }^{9}$.

Durkheim distingue entre lazos verdaderos y no verdaderos, tal como lo hace Rousseau y de acuerdo a un criterio que en lo sustancial no es muy diferente. Pues en última instancia, para ambos autores, aquello que decide sobre la verdad (o falsedad) del lazo es la capacidad (o incapacidad) del mismo para asociar las partes que componen el todo y, asimismo, mantener vivo el sentimiento de unidad del cuerpo social. Esto no es contradictorio respecto de lo que afirmamos hasta el momento. La teoría de Durkheim sobre el origen y la naturaleza del lazo social difiere en muchos aspectos de la de Rousseau. Lo que empezamos a detectar como resultado del análisis es que ambos autores comparten un mismo modelo de socialidad, una misma representación arquetípica de la relación social.

\section{DESENLACE}

Su modelo de socialidad es, precisamente, la sociabilidad o, mejor aún, lo que a partir de ahora y para evitar confusiones podriamos llamar la convivialidad. En efecto, tanto Rousseau como Durkheim tienden a representar el socius humano bajo la forma de relaciones positivas entre los individuos y la sociedad, o también, dicho en un lenguaje que comparten, bajo la forma de una relación verdadera entre las partes y el todo. Tomada como metáfora de la convivialidad, la figura del lazo social lleva estas significaciones mucho más allá de los discursos que la

\footnotetext{
${ }^{9}$ Para un análisis en profundidad de los elementos normativos presentes en la obra de Durkheim, ver Weiss (2010).
} 
volvieron célebre. Sin embargo, este no es más que uno de los aspectos sobre los que deseábamos llamar la atención. A continuación y para concluir, vamos a detenernos en aspectos menos visibles pero tan problemáticos como el anterior.

La metáfora del lazo social compromete en su uso cuestiones axiológicas, pero también epistemológicas y ontológicas. Sobre las primeras nos hemos detenido largamente. Sobre las otras, en cambio, sólo hemos hecho algunas indicaciones breves y más bien elípticas que ahora es preciso poner en evidencia. La inconveniencia de esta metáfora no reside únicamente en la fuerte carga valorativa que posee, sino también en el hecho de que su uso para mentar la existencia en común expresa un punto de vista cuyo horizonte insuperable es el individuo. Invariablemente, sea de manera natural o artificial, el lazo social se instaura entre individualidades constituidas. El lazo es lo que viene a poner en relación individuos que, según un razonamiento elemental, debemos presumir no solamente constituidos o individuados sino también separados, independientes unos de otros. El lazo se presenta una y otra vez como algo que adviene o sobreviene, como algo que le sucede a las individualidades aisladas. Esto quiere decir que la existencia del lazo supone necesariamente la existencia previa de los términos enlazados. El lazo social se deduce de una individualidad primera que de forma explícita o implícita siempre aparece como algo dado. El presupuesto general y mayormente impensado de esta categoría nodal es la preexistencia de los individuos respecto de la existencia social.

El mayor inconveniente que presenta semejante lógica de pensamiento es la clausura de cualquier alternativa a la oposición entre individuo y sociedad. Sea cual sea el punto de partida elegido, la noción de lazo social está supeditada a dicha oposición y no hace más que confirmarla. Aún más, creemos que esta noción impide pensar aquello que es condición de posibilidad de lo que habitualmente entendemos por individuo y sociedad, a saber, la relación en cuanto tal. Tanto para Rousseau como para Durkheim, la idea de relación es indisociable de la de lazo social, de modo tal que ni uno ni otro concibe el socius sin individuos presupuestos o preexistentes que la hagan posible. Paradójicamente, una expresión que funcionó a lo largo de la historia como la imagen convivial de la relación social, en verdad, no hizo otra cosa que subsumir la realidad relacional, que estrictamente hablando no es individual ni colectiva, a la realidad del individuo, del ente uno e 
indivisible que al menos desde Aristóteles sirve de modelo a la sustancia. Dicho en lenguaje aristotélico, el individuo es la "sustancia primera", el ente concreto que existe en sí mismo independientemente de cualquier otro ente. La sustancia siempre es sujeto, nunca predicado. Mientras que la relación es un "accidente" de la sustancia, es decir, algo que le ocurre al individuo.

Si lleváramos el problema que nos ocupa a este terreno aparentemente ajeno y anacrónico, se podría decir que el lazo social es accidental, no sustancial. Cuando Rousseau y Durkheim se refieren a la consistencia y a la durabilidad del lien social utilizando a veces las mismas palabras; cuando ponen a jugar los dos sentidos (el literal y el figurado) de la palabra lien, sucede que el vínculo inmaterial, espiritual o moral se vuelve indiscernible del lazo material. Una de las consecuencias de presentar el lazo social como si se tratara de una "cosa", es la de producir un efecto de exterioridad entre, por un lado, los individuos, y, por el otro, la ligadura que viene a unirlos. La unión de las partes que componen el todo depende de este elemento sumamente cambiante que tanto se anuda como se desanuda, cuando no se hace trizas. Según este criterio, lo social procede del afuera y permanece en el afuera. Es exterior o extranjero $y$, por lo tanto, es de otra naturaleza que las interioridades que relaciona. De este modo, se puede entender el lazo social como una suerte de mediación externa entre el adentro y el afuera. La metáfora del lazo confirma una y otra vez la vieja dualidad entre la interioridad individual y la exterioridad social. Reproduce la diferencia al mismo tiempo que parece superarla. Pero en realidad no hay superación ni relevo de ningún tipo. Sistemáticamente, lo individual queda del lado "de acá" y lo social del lado "de allá". El ser individual y el ser social se diferencian tanto como pueden hacerlo una sustancia y un accidente.

Por cierto, los autores considerados no suelen emplear este lenguaje ni son especialmente afectos a la especulación metafísica. Rousseau, por su lado, renuncia a conocer la naturaleza humana por considerarla una empresa prácticamente imposible. Sin embargo, no puede renunciar a adoptar un punto de vista sobre la constitución del hombre, aunque más no sea conjetural. El individualismo de Rousseau - por el que Durkheim lo criticará- es una de las marcas distintivas y más comentadas de su obra. Allí, el individuo es el punto de partida metodológico o epistemológico. Esto significa que todo se explica a partir de las fuerzas, las necesidades, los deseos y las capacidades individuales. Recordemos 
que el verdadero lazo social es la unión artificial y extrínseca entre los diferentes individuos. Pero también, y lo que es distinto, el individuo es el fundamento de la ontología que subyace a su teoría. Si ponemos a un lado la comunidad originaria por considerarla una hipótesis o un mito, lo que queda como realidad última de la existencia es el ser humano individual.

La crítica a Rousseau introducía un radical reposicionamiento y el argumento central de una nueva ciencia social. Podemos estar seguros de que la sociología de Durkheim no es individualista, pero esto no significa que este rechace todo individualismo. En este punto, se vuelve necesario distinguir entre, por una parte, el individualismo como método de explicación de los fenómenos sociales $y$, por otra, el "individualismo moral" (opuesto al "individualismo egoísta") en tanto sistema de creencias que asegura la armonía entre las partes y el todo en las sociedades modernas ${ }^{10}$. La crítica de Durkheim se dirige en particular a las perspectivas que intentan explicar la realidad social a partir de la realidad individual. El mentado sociologismo durkheimiano consiste básicamente en tomar a la sociedad como punto de partida metodológico. La tesis según la cual los fenómenos sociales se explican por causas sociales, y no individuales, es una constante de este pensamiento. La ciencia de la sociedad justifica esta posición epistemológica en el hecho de que la figura del individuo se fue construyendo a lo largo de la historia en lugares y momentos específicos. El individuo es un producto del conjunto de creencias y prácticas comunes que rigen en un espacio y un tiempo determinados. Por lo tanto, no puede servir de fundamento explicativo invariable de la vida social en su conjunto.

En Las reglas del método sociológico Durkheim reivindica una "sociología objetiva, específica y metódica" (1997: 14) contra toda concepción metafísica y contra toda especulación en general. Buena parte de su esfuerzo teórico y metodológico está encaminado a demostrar la cientificidad de la sociología y la autonomía de la misma respecto de otras disciplinas cercanas. Sin embargo, nada de esto nos impide reconocer en su empresa una deriva ontológica implícita y persistente. Durkheim reafirma una y otra vez que la naturaleza de la sociedad es diferente de la naturaleza de los individuos particulares y que para

\footnotetext{
${ }^{10}$ Sobre los distintos significados del término "individualismo" en la obra de Durkheim, ver Girola (1997).
} 
comprender esta diferencia es necesario distinguir entre los "estados de la conciencia colectiva", por un lado, y los "estados de la conciencia individual", por otro. En un caso se trata de "representaciones colectivas", en el otro de "representaciones individuales". Estos son los objetos específicos y relativamente independientes de la sociología y la psicología respectivamente. No obstante, he aquí toda la cuestión, unas y otras son representaciones, y en cuanto tales sólo pueden tener lugar a través de las conciencias individuales. Para conocer científicamente los hechos sociales, "hay que tomar en consideración el agregado en su totalidad. Es ese conjunto el que piensa, que siente, que quiere, aunque no pueda querer, sentir $u$ obrar sino por intermedio de las conciencias particulares" (Durkheim, 2000: 50). Aun si los hechos sociales difieren de los hechos psíquicos en aspectos decisivos, "[e]sto no significa que no sean, también ellos, psíquicos de alguna manera, puesto que todos consisten en modos de pensar o de actuar" (ibídem, 1997: 22).

El "principio fundamental" del método sociológico es "la realidad objetiva de los hechos sociales" (ibídem: 31), del cual se deriva la famosa regla que consiste en considerar los hechos sociales como cosas. Estos son "modos de actuar, de pensar y de sentir que presentan la propiedad notable de que existen fuera de las conciencias individuales" (ibídem: 39). Además de ser exteriores, los hechos sociales son coercitivos y colectivos. Durkheim califica a estos fenómenos como "sociales" y afirma que este calificativo "[I]es corresponde porque está claro que, no teniendo por sustrato al individuo, no pueden tener otro más que la sociedad" (ibídem: 41). Ahora bien, ¿la sociedad es verdaderamente el "sustrato", es decir lo que subyace a los hechos sociales? En realidad, el argumento desplegado por Durkheim habilita una interpretación en sentido contrario. La vida colectiva no se explica por la vida individual y, sin embargo, la presupondría desde el momento en que se reconoce el fundamento psicológico de esta teoría. El sustrato, el soporte o, para decirlo en los términos de la filosofía clásica, la sustancia que soporta accidentes, no sería la sociedad sino la psique del individuo ${ }^{11}$.

\footnotetext{
${ }^{11}$ Se podrian multiplicar las citas de textos anteriores y posteriores a Las reglas del método sociológico donde Durkheim sostiene el carácter psíquico de los fenómenos sociales. Asimismo, son numerosos los pasajes donde examina semejanzas y diferencias entre las representaciones individuales y colectivas. En Las reglas del método sociológico, allende la especificidad que le atribuye a cada una de estas representaciones, también reconoce la imposibilidad de contestar con seguridad a la pregunta por lo que habría de común entre ambas (1997: 24-25). Por lo demás, Durkheim se exonera a sí mismo y a su tiempo de
} 
La primacía del individuo es rechazada en el plano epistemológico pero subsistiría tácitamente en el plano ontológico, dando lugar a una serie de controversias a las que Durkheim no fue ajeno y que aún hoy se encuentran vigentes. Además, cabe destacar que esta ambigüedad es favorecida por una retórica recurrente donde los individuos aparecen, ora como condición, ora como resultado de la vida común. Por un lado, el todo es algo distinto y es algo más que la suma de sus partes. La sociedad tiene su propia naturaleza y detenta una supremacía moral sobre los miembros que la componen, de modo tal, que se les impone desde afuera y moldea sus modos de ser y de hacer. Por otro lado, el todo está formado por la unión de las partes constituyentes. La sociedad es una síntesis sui generis, es el producto que surge de la mezcla de las acciones individuales, confirmando así que la existencia de la sociedad depende de la existencia de los individuos. Esta ambigüedad que Durkheim fomenta consciente o inconscientemente es parte pregnante del problema del lazo social ${ }^{12}$.

Gracias al uso cada vez más generalizado, naturalizado y eventualmente neutralizado de esta metáfora, se corre el riesgo de olvidar que el lazo social es un problema, una suerte de nudo compacto que es necesario empezar a desanudar si lo que se pretende es descifrar tanto su genealogía histórica como una serie de implicaciones que lo atraviesan de punta a punta. El análisis que nos condujo hasta aquí no pretende otra cosa más que avanzar en la dirección de un tal desciframiento. Aunque sabemos que no es suficiente. En un movimiento desdoblado, diferenciado pero paralelo, habría que poder desanudar el nudo y desenlazar el lazo.

encontrar una respuesta a dicha pregunta cargándola a la cuenta del "futuro" y admitiendo que se trata de "un problema que pertenece más a la jurisdicción de la filosofía general y de la lógica abstracta que al estudio científico de los hechos sociales" (ibídem: 26-27).

${ }^{12}$ Resulta sorprendente que los intérpretes contemporáneos de Durkheim todavía contribuyan mediante sus afirmaciones a esta ambivalencia que sin duda nos aleja más de lo que nos acerca a una comprensión cabal de su teoría. Por ejemplo, la lectura de Bruno Karsenti (2006) expone con agudeza la concepción durkheimiana sobre la relación entre la sociedad y los individuos sin dejar de lado la dimensión ontológica del problema, la cual fue sistemáticamente negada por el propio Durkheim y por la inmensa mayoría de los comentaristas. Sin embargo, continúa reproduciendo a su manera la ambigüedad acerca de la relación de dependencia ontológica entre el plano del ser individual y el plano del ser social. 
El desenlace del lazo es el final de un recurso explicativo que aplaza indefinidamente la explicación de aquello que se obstina en designar $y$, en verdad, todavía no ha pensado más allá de los límites impuestos por el binarismo tradicional. Por esa razón, el desenlace del lazo es también una tentativa de comienzo o recomienzo. Es la posibilidad de pensarnos, en primer lugar, no como individuos que entran en relación y de ese modo hacen sociedad, sino más bien como individuos sociales que existen según la modalidad relacional. El conjunto de relaciones sociales al que denominamos sociedad no puede ser el resultado de individuos aislados que se han puesto a sí mismos en relación. La sociedad no es un hecho nuevo conforme a sucesos previos que estarian representados por los individuos originalmente separados, en la misma medida que no es el efecto de la mezcla de elementos que hasta el momento de la asociación debemos suponer puros. Imaginar que hay algo así como sustancias individuales que anteceden a la sociedad es tan quimérico como imaginar una pretendida sustancia social anterior a los individuos. Es a partir de las relaciones que las categorias de individuo y sociedad y las realidades que ellas representan cobran sentido, no a la inversa. Por eso mismo es que nunca entramos en relación, sino que somos o estamos siempre ya en relación. Dicho de otra forma, son las relaciones histórico-sociales las que nos hacen ser lo que somos. Contra la idea obcecada de lazo social, solidaria como es de una serie de oposiciones extenuadas pero aún imperantes, aqui se afirma el carácter constitutivo de la relación.

Darle a la relación un sentido constitutivo tiene consecuencias considerables que aquí no podemos tratar más que de forma tangencial. Nos limitaremos por el momento a hacer tres breves consideraciones. En primer lugar, aquí entendemos la relación en el sentido amplio y esencialmente indeterminado del término. Es decir, que no hay ninguna relación social particular que sea condición o fundamento de las demás. Desde el momento en que se excluye toda jerarquía entre las relaciones sociales, caracterizaciones del tipo relaciones verdaderas o falsas pierden pertinencia. Lo que llamamos "relación" no debe ser automáticamente identificado con "sociabilidad", "solidaridad", "cohesión", "unión", "orden social", ni con sus contrarios. Relación es la condición misma de cualquier forma imaginable de vida en común, tanto las buscadas o deseadas como las indeseadas. En segundo lugar, y a fin de evitar un acercamiento puramente especulativo a esta 
perspectiva, es necesario reconsiderar el estatuto epistemológico de las relaciones sociales. Con respecto a esta enorme cuestión, resulta de gran interés el planteo de Philippe Corcuff (2013) sobre el "relacionalismo metodológico" como vía alternativa al individualismo y al holismo. Este planteo toma a las relaciones sociales como "entidades primordiales" y a "los actores individuales y las formas colectivas como entidades secundarias" (ibídem: 27). En tercer y último lugar, la relación tiene un sentido ontológico insoslayable. Concebir una ontología basada en las relaciones sociales requiere para empezar que situemos la noción misma de relación en un lugar distinto al que ocupó tradicionalmente en los discursos filosóficos y científico-sociales. Hay que entender, pues, que la relación no es un "accidente" posterior y secundario respecto a la "sustancia primera", ni es un vínculo entre términos desvinculados y ya constituidos. Desde un punto de vista ontológico, la relación es primera, lo que equivale a afirmar el origen necesariamente plural de las existencias singulares. En definitiva, lo que nos interesa es cuestionar tanto el sustancialismo que hace primar al ser individual como a aquel que hacer primar al ser colectivo $y$, en su lugar, colocar al ser relacional entendido como aquello que no puede ser reducido a ni apropiado por ningún tipo de sustancia absoluta ${ }^{13}$.

Esta afirmación nos pone en camino hacia la construcción de otro concepto de socialidad que aquel comprendido en los alegatos del lazo social. Pero debe reconocerse a su vez que esta tarea exige una crítica sostenida de los binarismos que organizan nuestras formas de pensar lo social. En suma, hay que deconstruir para construir. Para ser consecuentes con este programa de investigación, habría que avanzar en la deconstrucción del vasto sistema de oposiciones presente tanto en los discursos que venimos de analizar como en tantos otros que todavía hoy se muestran inconmovibles. Todo hace pensar que la posibilidad de construir algo nuevo en el corazón de lo viejo depende, en gran parte, de nuestra capacidad para conmover los fundamentos de un sistema que contribuye a la naturalización de lo social y a la prescripción de formas predeterminadas de socialidad, formas supuestamente verdaderas o auténticas de coexistencia.

El desenlace del lazo anuncia, pues, el final de un cierto régimen de pensamiento social. Es el anuncio de una mutación en el modo de

\footnotetext{
${ }^{13}$ Para un tratamiento más amplio del problema de la relación, me permito remitir a mis trabajos (Alvaro, 2012, 2015, 2016).
} 
concebir eso mismo que la expresión "lazo social" sugiere allí donde todavía encuentra lugar. Pero, sobre todas las cosas, hay que evitar asociar esta declaración con los argumentos apocalípticos en boga. El desenlace del lazo no declara el fin de las grandes tradiciones intelectuales que emplearon esta metáfora a lo largo de la historia como tampoco declara la desaparición de tal o cual organización social ni la muerte de lo social en cuanto tal. Nada de eso. Acaso si algo encuentra aquí su término es nuestra confianza ciega en una metáfora que acaba por subordinar la multiplicidad de la realidad relacional a la unicidad de la realidad sustancial, hundiendo en la oscuridad aquello que precisamente quisiera sacar a la luz. De lo que aquí se trata, entonces, es de una invitación a transitar un camino ya abierto pero en buena medida aún inexplorado, un camino que sin renunciar enteramente a la herencia recibida asume la tarea exigente de pensar la socialidad sin ataduras.

\section{BibLiOgRAFÍA}

Alvaro, D. (2012). Marx y la ontología de lo común. Nómadas, 36(II), 13-29.

Alvaro, D. (2015). Ontologías del ser social (Lukács, Gould, Negri, Hardt, Balibar). Nómadas, 45(I), 23-53.

Alvaro, D. (2016). Lo transindividual: de Simondon a Marx. TRANS/FORM/AÇÃO, 39(4), 153-172.

Belton-Chevallier, L. (2009). Mobilités et lien social: sphères privée et professionnelle à l'épreuve du quotidien. Thèse de Doctorat de I'Université Paris-Est. Disponible en: https://halshs.archivesouvertes.fr/tel-00545636/document.

Bernardi, B. (2006). La fabrique des concepts: recherches sur l'invention conceptuelle chez Rousseau. Paris: Honoré Champion Éditeur.

Bouvier, P. (2005). Le lien social. Paris: Gallimard.

Corcuff, Ph. (2013). Las nuevas sociologías. Principales corrientes y debates, 1980-2010. Buenos Aires: Siglo XXI.

Durkheim, É. (1970). Les études de science sociale. In La science sociale et I'action (pp. 184-214). Paris: PUF.

Durkheim, É. (1975a). La science positive de la morale en Allemagne. In Textes. 1. Éléments d'une théorie sociale (pp. 267-343). Paris: Minuit.

Durkheim, É. (1975b). Introduction à la sociologie de la famille. In Textes 3. Fonctions sociales et institutions (pp. 9-34). Paris: Minuit.

Durkheim, É. (1997). Las reglas del método sociológico. México: FCE. 
Durkheim, É. (2000). Representaciones individuales y representaciones colectivas. In Sociología y filosofía (pp. 27-58). Madrid: Miño y Dávila.

Durkheim, É. (2001). El contrato social, de Rousseau (1918). In Montesquieu y Rousseau. Precursores de la sociología (pp. 85-146). Madrid: Miño y Dávila.

Durkheim, É. (2003). Lecciones de sociología. Fisica de las costumbres y del derecho y otros escritos sobre el individualismo, los intelectuales y la democracia. Buenos Aires: Miño y Dávila.

Durkheim, É. (2008). La división del trabajo social. Buenos Aires: Gorla.

Genestier, P. (2006). L'expression 'lien social': un syntagme omniprésent, révélateur d'une évolution paradigmatique. Espaces et sociétés, 126, 19-34.

Girola, L. (1997). El individualismo según Durkheim. Sociológica, 34, 69-88.

Godicheau F., \& Sánchez León, P. (Eds.). (2015). Palabras que atan. Metáforas y conceptos del vínculo social en la historia moderna y contemporánea. Madrid: FCE-Université Bordeaux Montaigne.

Karsenti, B. (2006). La société en personnes. Études durkheimiennes. Paris: Economica.

Lepan, G. (2014). L'amitié et l'attachement. In B. Bachofen, B. Bernardi, A. Charrak \& F. Guénard (Dir.), Philosophie de Rousseau (pp. 319-335). Paris: Classiques Garnier.

Lukes, S. (1984). Émile Durkheim. Su vida y su obra. Madrid: Siglo XXI.

Paugam, S. (2008). Le lien social. Paris: PUF.

Rossi, M. A. (Comp.). (2015). El lazo social desde la filosofía política. Buenos Aires: Grama.

Rousseau, J.-J. (1993). El contrato social. Barcelona: Altaya.

Rousseau, J.-J. (2004). El contrato social o Ensayo sobre la forma de la República [Manuscrito de Ginebra]. Deus Mortalis, 3, 549-608.

TLFi - Trésor de la Langue Française informatisé, ATILF, CNRS, Université de Lorraine. Disponible en http://atilf.atilf.fr/.

Weiss, R. A. (2010). Émile Durkheim e a Fundamentação Social da Moralidade. Tese de Doutorado de Universidade de São Paulo. Disponible

en: www.teses.usp.br/teses/disponiveis/8/8133/tde.../2010_RaquelAndr adeWeiss.pdf. 